\title{
Multivariate standard addition for the analysis of overlapping voltammetric signals in the presence of matrix effects: application to the simultaneous determination of hydroquinone and catechol
}

Kassandra Martínez, Cristina Ariño, José Manuel Díaz-Cruz*, Núria Serrano and Miquel Esteban

Departament d'Enginyeria Química i Química Analítica. Facultat de Química. Universitat de Barcelona. Martí i Franquès, 1-11, 08028 Barcelona (Spain).

*Corresponding author. e-mail: josemanuel.diaz@ub.edu, Phone: (+34) 934021796.

\section{ABSTRACT}

A multivariate version of the classical univariate standard addition method is tested as a proof of concept for the voltammetric analysis of complex samples generating overlapped signals in the presence of significant matrix effects. The proposed strategy applies a multivariate calibration method such as partial least squares (PLS) to the full voltammograms measured for the sample alone and after combined additions of a series of standard solutions (one for every analyte). Then, a calibration model is built and further applied to the prediction of the concentration added to a blank, i.e., a full voltammetric signal measured in the absence of analytes. The absolute value of such predicted concentration is taken as the concentration of the analyte in the sample. The method has been successfully tested in different natural water samples spiked with hydroquinone and catechol and appears to be a promising tool for the analysis of overlapped signals in complex matrices.

keywords: multivariate standard addition; partial least squares (PLS) calibration; voltammetry; hydroquinone; catechol; matrix effects. 


\section{Introduction}

Calibration is an essential aspect of instrumental analysis. In the case of univariate responses, external standard and standard addition are among the most popular calibration methods [1, 2]. The external standard method builds a calibration plot with the responses of a series of standards and uses the response of the sample to obtain the concentration of the analyte in the sample by interpolation. In contrast, the standard addition method constructs a calibration plot by registering the responses of the sample before and after successive additions of a standard solution of the analyte and obtains the analyte concentration in the sample by extrapolation to a zero response value. This method is particularly recommended when matrix problems exist and it is especially useful in electroanalysis.

The application of these classical strategies in univariate analysis is quite straightforward even in the presence of different analytes as far as their responses do not interfere with each other. However, this situation is not always fulfilled in spectroscopic or electroanalytical determinations. Quite often, they suffer from interactions between species, signal overlapping or matrix effects hindering the application of these methodologies. Then, multivariate analysis has to be carried out and chemometric techniques are required for the data treatment [3-5]. Among these, partial least squares (PLS) calibration is especially versatile and robust, but it is essentially an external calibration method which requires the use of a similar chemical matrix in the solutions of the standards and the samples.

Concerning to voltammetric measurements, standard addition has been for a long time the preferred calibration method, especially when complex samples are analysed [6]. This is because voltammograms are very sensitive to matrix effects and standard addition allows one to work in the presence of just the same matrix when measuring both samples and standards. Although voltammetry, by itself, is a multivariate measurement, if the signal of the analyte is well-defined and is not overlapped to other signals, its limiting current, its peak height or its peak area are just single numbers which can be taken instead of the full voltammogram to carry out an univariate description of the system and, therefore, to apply the classical standard addition method [6]. If different analytes are present in the sample producing perfectly resolved signals, additions of a solution containing all analytes can be made and separated plots can be drawn for every analyte representing the corresponding limiting currents, peak heights or peak areas versus the added concentration.

Unfortunately, this is not always the case. Quite often, voltammetric signals are strongly overlapped with each other and it is necessary to analyse the full voltammograms to guess the 
contribution of each analyte to the overall reponse. Thus, it would be necessary a multivariate extension of the classical standard addition method to take into account the whole voltammetric signal and not only the limiting current, the peak height or the peak area.

In the early years of Chemometrics, Kowalski and coworkers [7-9] had already proposed a multivariate version of the standard addition method, called generalised standard addition method (GSAM), which was successfully applied to data obtained by UV-vis spectrophotometry [10], inductively coupled plasma spectroscopy [11, 12] and anodic stripping voltammetry [13]. The method is based on the classical least-squares calibration (CLS) of a 'signal increase' matrix obtained by subtracting the spectra of the sample from the original data matrix containing all the spectra measured after successive additions of standards. Such matrix is divided by that containing the corresponding added concentrations to yield a sensitivity matrix that can be applied to the sample spectra to predict the concentration of all the analytes considered. Although the general formulation of the method also considered quadratic and cubic responses, experimental applications were restricted to the linear case. Later, a PLS version of GSAM was proposed [14] in order to deal with more intricate samples and/or higher noise. Although GSAM strategy was frequently used in the 1980's, its popularity decayed along the 1990's and the focus of multivariate standard addition moved to the study of second order data, mostly obtained with spectroscopic techniques [15-18].

More recently (2012), Melucci and Locatelli proposed a different and valuable point of view about multivariate standard additions in voltammetry [19]. Unlike GSAM approach, which operates with transformed data (original signals minus sample signals), these authors directly apply PLS to the original voltammograms of the sample before and after the additions in order to build a calibration model referred to added concentrations. Then, the model is applied to a blank voltammogram (i.e., measured in a solution containing the same matrix as before, but without analyte) to predict by extrapolation the concentration of the analyte in the sample. This PLS extrapolation approach was tested with synthetic solutions and real samples containing $\mathrm{Pb}(\mathrm{II})$-ions. In all cases, a single, perfectly defined peak of $\mathrm{Pb}(\mathrm{II})$ was obtained and this allowed the authors to compare the results achieved by using univariate data (the heights of the peaks) and multivariate data (the full voltammograms). As both kinds of results were fully consistent, they concluded that multivariate calibration via extrapolation to a blank signal was a promising strategy.

Nevertheless, multivariate calibration is seldom used to determine just one analyte producing a single peak. In this situation, univariate calibration is a much better choice. Multivariate 
methods like PLS are normally applied to strongly overlapped signals in the presence of intricate baselines. This is why in this work we try to extend the proof of concept proposed in ref. [19] to multicomponent analysis in the presence of overlapped signals. For this purpose, we have selected two dihydroxybenzene isomers, hydroquinone and catechol, which show a clear overlapping of their oxidation signals in voltammetric measurements [20-23]. In the last years, many efforts have been devoted to develop modified electrodes able to increase the separation between both peaks [20-22] but, as far as we know, a perfect resolution of both signals has not been achieved yet. In contrast, the use of relatively simple electrodes combined with a common multivariate calibration method like partial least squares (PLS) allowed a satisfactory determination of both analytes [23]. Then, we have taken as a reference the experimental methodology used in [23] but, in order to enhance the matrix effects, we have considered not only synthetic water solutions, but also natural waters of different origin spiked with both analytes.

\section{Theory}

Figure 1 compares the main features of the classical, univariate standard addition method (Figure 1a) with these of the multivariate methodology tested in the present work (Figure 1b). In both cases, the additions of known concentrations of the analyte produce a series of responses which are used to build a calibration model. In the univariate situation, the model is just the equation of a line (intercept and slope), whereas in multivariate calibration, the PLS model is more complicated and involves several matrices. When more than one analyte is determined, a separated model can be made for every analyte (PLS1) or a single model can be used for all analytes (PLS2). In both Fig. 1a and Fig. 1b the response of the sample (a black point and a thick curve, respectively) determine the origin of the added concentration axis. The corresponding models are extrapolated until they reach the response expected or measured for a blank solution (a zero value or a blank voltammogram). Then, a negative concentration is obtained which, once the sign is changed, yields the original concentration of the analyte in the sample. In Figure 1b, a PLS1 calibration is depicted, which means that only the concentration of one of the analytes is determined. In the case of PLS2, two or more added concentration axes would be present (impossible to represent in 3D) and the extrapolation of the model would extend along all axes to produce several negative concentration values, one for each analyte. Figure $1 c$ shows a typical predicted versus reference plot of PLS where the predicted concentrations are plotted as a function of the real values and the resulting points 
are compared with the ideal line of zero intercept and unity slope corresponding to a 'perfect' calibration. When multivariate standard addition is used, the points of the sample and the additions are in the positive-positive quadrant while the predictions of the original sample concentration are in the negative-negative quadrant. All these points have to be considered to evaluate the quality of the calibration by using parameters such us the determination coefficient $\mathrm{R}^{2}$ or the root mean squares error of calibration, cross-validation and prediction (RMSEC, RMSECV and RMSEP, respectively) [3].

Figure 2 compares different strategies to carry out the additions. When the signals of two analytes, A and B, are well resolved (Figure 2a), the univariate approach can be used by means of the peak heights of both signals. Then, additions can be made of a solution including known concentrations of both $A$ and $B$, so that every addition increases both peaks and provides two new values of peak height to be included in the respective, separated calibration plots. In contrast, if the peaks of $A$ and $B$ overlap with each other (Figure $2 b$ ), the multivariate approach has to be used and then the PLS analysis of the data generated by simultaneous additions of $A$ and $B$ is not able to distinguish the contribution of every analyte to the signal, since the evolution of the respective signals along the calibration is the same. More convenient is to carry out additions of individual solutions of A and B separately, so that PLS method can better resolve the contributions of $\mathrm{A}$ and $\mathrm{B}$. Among the possible experimental designs, Figure $2 \mathrm{C}$ shows an alternating addition of both $A$ and $B$ solutions, whereas Figure $2 \mathrm{~d}$ shows a series of additions of A followed by a series of additions of B. In the present work, some preliminary tests with synthetic mixtures of hydroquinone and catechol suggested that the design in Figure $2 c$ was slightly better, since it generates a more homogeneous distribution of points in the calibration plots and keeps a similar concentration ratio between both analytes along the full set of additions. Therefore, alternating additions of hydroquinone and catechol were used in all the experiments described below.

\section{Materials and methods}

\subsection{Chemicals and reagents}

Hydroquinone was obtained from Sigma-Aldrich (St. Louis, MO, USA) and catechol was provided by Honeywell-Fluka (Morristown, NJ, USA). Phosphate buffer solution (PBS) at pH 7.0 was prepared by mixing the suitable amounts of $0.1 \mathrm{~mol} \mathrm{~L}^{-1} \mathrm{NaH}_{2} \mathrm{PO}_{4}$ and $0.1 \mathrm{~mol} \mathrm{~L}^{-1} \mathrm{Na}_{2} \mathrm{HPO}_{4}$, both provided by Scharlab (Barcelona, Spain). All chemicals used were of analytical reagent grade, and the solutions were prepared in ultrapure filtered water obtained from Milli-Q plus 
185 system (Millipore, Milford, Massachusetts, USA). Hydroquinone and catechol solutions were prepared in free oxygen media and stored in the dark at $4^{\circ} \mathrm{C}$ to prevent oxidation.

In order to study the effect of the matrix on the experiments, a buffer solution with and without a high salt content $\left(\mathrm{NaCl} 0.5 \mathrm{~mol} \mathrm{~L}^{-1}\right)$ and different Ebro River water samples were used. The river samples were collected in Flix (Tarragona, Spain) close to a chlor-alkali plant and in Deltebre (Tarragona, Spain) with a high content of salt (Deltebre is located in the mouth of the river). Finally, a certified wastewater sample (SPS-WW2 from Spectrapure Standards AS, Oslo, Norway) was also employed. All these matrices were spiked with hydroquinone and catechol.

\subsection{Instrumentation}

A $\mu$ Autolab System Type III (EcoChemie, Netherlands) attached to a Metrohm 663VA Stand (Metrohm, Switzerland) and a personal computer with GPES4.9 Software (EcoChemie, Netherlands) was used to perform voltammetric experiments. A combined redox electrode (Crison, Spain) was used as combined reference $\left(\mathrm{Ag} / \mathrm{AgCl} 3 \mathrm{~mol} \mathrm{~L}^{-1} \mathrm{KCl}\right)$ and auxiliary (Pt wire) electrode. The working electrode was a graphene screen-printed electrode (SPE) with $4 \mathrm{~mm}$ diameter provided by Dropsens (Oviedo, Spain) (ref. DRP-110GPH). The screen-printed electrode was connected to the Autolab System by means of a flexible cable (ref. CAC, DropSens). Differential pulse voltammograms (DPV) were recorded from $-0.2 \mathrm{~V}$ to $0.9 \mathrm{~V}$ by using a step potential of $0.005 \mathrm{~V}$, a pulse amplitude of $0.05 \mathrm{~V}$ and a pulse time of $50 \mathrm{~ms}$. Ionic strength and $\mathrm{pH}$ were controlled in all measurements with $0.1 \mathrm{~mol} \mathrm{~L}^{-1} \mathrm{PBS}$ at $\mathrm{pH}=7$. All measurements were carried out in a glass cell at room temperature $\left(20^{\circ} \mathrm{C}\right)$ after oxygen removal. A Crison Micro pH2000 pH-meter (Crison, Spain) was used for pH measurement.

\subsection{Procedures}

Voltammetric analysis of samples consisted of three steps. First, $25 \mathrm{~mL}$ of the blank solution (containing the matrix of the sample in $0.1 \mathrm{~mol} \mathrm{~L}^{-1} \mathrm{PBS}$ at $\mathrm{pH}=7$ ) were placed in the cell and measured by DPV. Second, the blank was replaced by $25 \mathrm{~mL}$ of the sample (containing the analytes) and a new measurement was done. Third, successive additions of standard solutions of hydroquinone and catechol were carried out and new DPV measurements were done after every addition. Different addition strategies were tested, which are described and compared in the Results and Discussion section.

\subsection{Data treatment}


Data treatment was done inside Matlab ${ }^{\circledR}$ environment [24] by means of the package PLS_Toolbox by Eigenvector [25]. This includes the integration of individual voltammograms into data matrices, the baseline corrections (for both samples and blanks) according to Whittaker [26-27] and automatic weighted least squares (AWLS) procedures [25] and the application of PLS calibration.

\section{Results and discussion}

In the Theory section, Figure 1 summarized the proposed strategy of multivariate standard addition and Figure 2 compared different ways to carry out the successive additions of the standard solutions of the analytes to the sample. In order to test the validity of this approach, several synthetic and natural samples spiked with hydroquinone and catechol were submitted to additions of both analytes. After some preliminary tests, the method in Figure $2 \mathrm{c}$ was preferred to that in Figure $2 \mathrm{~d}$, since it provided more reliable results, probably because it keeps a more balanced weight of both signals in the multivariate calibration. Figure 3 shows the voltammograms measured in the different spiked samples before and after successive additions of hydroquinone and catechol, in a way similar to the scheme in Figure 2c. The baseline corresponding to the blank (i.e., the matrix of the sample before it was spiked with analytes) was also measured and it is shown in black colour in the plots of Figure 3 . As it can be seen, blank baselines are quite different in the selected matrices. It must be stressed the relevance of this blank voltammogram, which is the target of PLS extrapolation. The voltammogram of the originally spiked sample (in green colour) is repeated three times. Then, groups of two successive additions of hidroquinone (red voltammograms) and catechol (blue voltammograms) are made in an alternating way. Figure 3 and Table 1 show that, although the difference in the peak potentials remains practically constant along the titration (ca. $100 \mathrm{mV}$ ), the relative evolution of the heights of both hidroquinone and catechol peaks is quite different in the matrices studied (their current ratios range from 1.25 in the MilliQ water to 1.57 in the Deltebre water).

In the chemometric analysis of the data sets shown in Figure 3 baselines of both samples and blanks were corrected using different strategies and the best results were obtained with the Whittaker and automatic weighted least squares (AWLS) methods [19]. Figure 4 shows the effects of both types of baseline correction on a set of original DP voltammograms. As for the number of latent variables (LV), it was determined by cross validation (CV) using the 'leave one out' approach [25]. In most of the samples only two or three latent variables were found by CV 
and, as discussed later, such a small number of LV was able to provide an accurate PLS calibration. Taking into account that the number of (spiked) analytes is two, these facts suggest an essentially linear behaviour of the voltammetric data acquired in these samples. In contrast, the certified residual water sample appeared to be more complex and the analysis of the corresponding voltammograms required five latent variables according to $\mathrm{CV}$. This could be due to the presence of other electroactive substances, to matrix effects and/or to deviations from linearity. Anyway, these five LV were also able to provide a satisfactory PLS calibration.

Figure 5 shows, as an example, the results of the application of PLS1 to the set of voltammograms measured in the certified wastewater sample (Figure 3e). Both a PLS model for hydroquinone (Figure 5a) and a PLS model for catechol (Figure 5b) with five LV each were built using the voltammograms of the spiked sample before and after all the alternating standard additions of hydroquinone and catechol. The root mean square error of calibration (RMSEC) was reasonably small in both cases $(0.609$ and 0.215$)$ and moderately increased, as expected, in cross validation (RMSECV of 1.620 and 0.737 ). The bias was also good in both calibration (0.0001 and 0.001$)$ and cross validation $(-0.416,-0.154)$. The plots of predicted versus real concentration (black points) show a perfectly linear behaviour, with $\mathrm{R}^{2}$ values approaching 1 in both calibration (0.998 and 1.000) and cross-validation (0.991 and 0.998). Moreover, they are very close to the ideal line with zero intercept and slope unity (in green colour). When these PLS1 models are applied to the voltammogram measured for the blank, the prediction is a negative value denoted by a red point in the graph. The negative sign is due to the fact that the axes are referred to added concentration, as depicted in Figure 1c. As Figures $5 \mathrm{a}$ and $5 \mathrm{~b}$ show, this prediction is very good since it is really close to the 'ideal' green line mentioned above. The reasonable root mean square errors (RMSEP of 0.226 and 0.226 ) and bias $(-0.226$ and -0.226$)$ confirm this perception.

Table 2 summarizes the results obtained in the application of the PLS method (in both PLS2 and PLS1 modes) to all data sets shown in Figure 3. It contains not only the predicted concentrations of both hidroquinone and catechol, but also the error of the predictions, the coefficient of determination of the prediction plot $\left(\mathrm{R}^{2}\right)$ and the root mean squares error (RMSE) values for all the analysed samples. The data in Table 2 suggest that PLS1 and PLS2 models produce relatively similar results. Hydroquinone predictions are a bit poor in the synthetic samples with only buffer or with $\mathrm{NaCl} 0.5 \mathrm{~mol} \mathrm{~L}^{-1}$, but the rest of hydroquinone predictions and all catechol predictions are quite good, with errors always lower than $10 \%$ and in most cases lower than $5 \%$. In general terms, errors are lower for catechol maybe because the signal of hydroquinone is placed on a potential region where the baseline presents a high 
current on the left, followed by a broad peak and a drastic decrease of the signal on the right, whereas the peak of catechol arises in a less problematic region of the baseline (Figure 3). Anyway, results are enough reliable to confirm the suitability of the proposed multivariate standard addition approach in the analysis of water samples with different matrices.

\section{Conclusions}

The obtained results indicate that the proof of concept by Melucci and Locatelli (i.e., the PLS extrapolation of full voltammograms towards the blank signal) can be extended from a friendly situation consisting on a single, well-defined peak to a more complicated yet realistic case involving a set of overlapping signals in the presence of complex matrices. In this extended methodology, the alternating addition of the different analytes plays a key role.

It must be pointed out that the present proof of concept has been tested with synthetic or spiked sample solutions where it was perfectly possible to measure the voltammogram of the blank and use it to extrapolate PLS models. However, in real situations involving unknown and complex matrices containing significant concentrations of the analytes it may be difficult to obtain a true blank solution, since analytes should be removed keeping the matrix unaffected. A possible solution to this problem could be using unpolluted samples of similar origin or playing with the deposition time in methods involving accumulation of the analytes.

\section{ACKNOWLEDGMENTS}

This work is supported by the Generalitat of Catalonia (Project 2014SGR269). 


\section{REFERENCES}

[1] D.C. Harris, Quantitative Chemical Analysis, $7^{\text {th }}$ Ed., W.H. Freeman and Company, New York, USA, 2007, pp. 69-90.

[2] J.N. Miller, J.C. Miller, Statistics and Chemometrics for Analytical Chemistry, $6^{\text {th }}$ Ed., Pearson, Essex, UK, 2010, pp. 110-151.

[3] S.D. Brown, R. Tauler, B. Walczak Eds., Comprehensive Chemometrics, Elsevier Science, Amsterdam, The Netherlands, 2009.

[4] M. Esteban, C. Ariño, J.M. Díaz-Cruz, M.S. Díaz-Cruz, R. Tauler, Multivariate Curve Resolution with Alternating Least Squares Optimization: A Soft-Modelling Approach to Metal Complexation Studies by Voltammetric Techniques. Trends Anal. Chem., 19 (2000) 49-61.

[5] M. Esteban, C. Ariño, J.M. Díaz-Cruz, Chemometrics for the analysis of voltammetric data, Trends Anal. Chem. 25 (2006) 86-92.

[6] J. Wang, Analytical electrochemistry, $3^{\text {rd }}$ Ed., John Wiley \& Sons, New York, USA, 2006.

[7] B.E. Saxberg, B.R. Kowalski, Generalized standard addition method, Anal. Chem. 51 (1979) 1031-1038.

[8] C. Jochum, P. Jochum, B.R. Kowalski, Error propagation and optimal performance in multicomponent analysis, Anal. Chem. 53 (1981) 85-92.

[9] J.H. Kalivas, Precision and stability in the generalized standard addition method, Anal. Chem. 55 (1983) 565-567.

[10] J.H. Kalivas, B.R. Kowalski, Automated multicomponent analysis with correction for interferences and matrix effects, Anal. Chem. 55 (1983) 532-535.

[11] J.H. Kalivas, B.R. Kowalski, Generalized standard addition method for multicomponent instrument characterization and elimination of interferences in inductively coupled plasma spectroscopy. Anal. Chem. 53 (1981) 2207-2212.

[12] E.A.G. Zagatto, A.O. Jacintho, F.J. Krug, B.F. Reis, Flow injection systems wiht inductivelycoupled argon plasma atomic emission spectrometry: Part 2. The generalized standard addition method, Anal. Chim. Acta 145 (1983) 169-178.

[13] R.W. Gerlach, B.R. Kowalski, The generalized standard addition method: intermetallic interferences in anodic stripping voltammetry, Anal. Chim. Acta 134 (1982) 119-127.

[14] I.E. Frank, J.H. Kalivas, B.R. Kowalski, Partial least squares solutions for multicomponent analysis, Anal. Chem. 55 (1983) 1800-1804.

[15] V.A. Lozano, G.A. Ibáñez, A.C. Olivieri, A novel second-order standard addition analytical method based on data processing with multidimensional partial least-squares and residual bilinearization, Anal. Chim. Acta 651 (2009) 165-172.

[16] V.A. Lozano, R. Tauler, G.A. Ibáñez, A.C. Olivieri, Standard addition analysis of fluoroquinolones in human serum in the presence of the interferent salicylate using lanthanide-sensitized excitation-time decay luminescence data and multivariate curve resolution, Talanta 77 (2009) 1715-1723.

[17] E. Peré-Trepat, S. Lacorte, R. Tauler, Alternative calibration approaches for LC-MS quantitative determination of coeluted compounds in complex environmental mixtures using multivariate curve resolution, Anal. Chim. Acta 595 (2007) 228-237.

[18] A. Afkhami, M. Abbasi-Tarighat, M. Bahram, H. Abdollahi, A new strategy for solving matrix effect in multivariate calibration standard addition data using combination of $\mathrm{H}$ point curve isolation and H-point standard addition methods, Anal. Chim. Acta 613 (2008) 144-151. 
[19] D. Melucci, C. Locatelli, Multivariate calibration in differential pulse stripping voltammetry using a home-made carbon-nanotubes paste electrode. J. Electroanal. Chem. 675 (2012) 25-31.

[20] L. Tang, Y. Zhou, G. Zeng, Z. Li, Y. Liu, Y. Zhang, G. Chen, G. Yang, X. Lei, M. Wu, A tyrosinase biosensor based on ordered mesoporous carbon-Au/L-lysine/Au nanoparticles for simultaneous determination of hydroquinone and catechol. Analyst 138 (2013) 3552-3560.

[21] T. Gan, J. Sun, K. Huang, L. Song, Y. Li, A graphene oxide-mesoporous $\mathrm{MnO}_{2}$ nanocomposite modified glassy carbon electrode as a novel and efficient voltammetric sensor for simultaneous determination of hydroquinone and catechol. Sens. Actuators $B$ Chem. 177 (2013) 412- 418.

[22] Z. Li, Y. Yue, Y. Hao, S. Feng, X. Zhou, X., A glassy carbon electrode modified with cerium phosphate nanotubes for the simultaneous determination of hydroquinone, catechol and resorcinol. Microchimica Acta, 185 (2018), 215.

[23] M. Aragó, C. Ariño, A. Dago, J.M. Díaz-Cruz, M. Esteban, Simultaneous determination of hydroquinone, catechol and resorcinol by voltammetry using graphene screen-printed electrodes and partial least squares calibration. Talanta 160 (2016) 138-143.

[24] Matlab, version R2009b Ed., Mathworks Inc.: Natick, MA, USA, 2009.

[25] PLS Toolbox version 7.8.2, Eigenvector Research Inc., Wenatchee, USA, 2014.

[26] E.T. Whittaker, On new method of graduation Proc. Edinburgh Math. Soc., 41 (1923), pp. 63-75.

[27] P.H. Eilers, A perfect smoother, Anal. Chem. 75 (2003) 3631-3636. 


\section{Table 1}

Differences in the peak potentials $(\triangle \mathrm{E})$ and ratios between the peak heights $(\mathrm{R})$ of the DPV signals of catechol $(\mathrm{CC})$ and hydroquinone (HQ) in the experimental runs shown in Figure 3. The currents considered to compute the ratio $\mathrm{R}$ have been taken in the original data matrix from the highest peak current to the baseline of the blank.

\begin{tabular}{|l|l|l|}
\hline Sample & $\Delta E(m V)=E_{p, C C}-E_{p, H Q}$ & $R=I_{p, C c} / I_{p, H Q}$ \\
\hline milliQ water & 103 & 1.25 \\
\hline NaCl 0.5 mol L-1 & 100 & 1.53 \\
\hline Flix water & 101 & 1.40 \\
\hline Deltebre water & 102 & 1.57 \\
\hline certified wastewater & 101 & 1.52 \\
\hline
\end{tabular}




\section{Table 2}

Results of PLS calibration by multivariate standard addition applied to the determination of hydroquinone (HQ) and catechol (CC) in different spiked samples. Baseline correction methods used (Whittakker and automatic weighted least squares filters) were available inside PLS-Toolbox [25].

\begin{tabular}{|c|c|c|c|c|c|c|c|c|c|c|}
\hline \multicolumn{11}{|c|}{ Hydroquinone (HQ) } \\
\hline \multirow[t]{2}{*}{ Sample } & \multirow{2}{*}{$\begin{array}{l}\text { Baseline }{ }^{a} \text {, } \\
\text { Model }\end{array}$} & \multirow{2}{*}{$\begin{array}{c}\text { Num. } \\
\text { LV }\end{array}$} & \multicolumn{2}{|c|}{$\mathrm{C}_{\mathrm{HQ}}(\mathrm{mg} / \mathrm{L})$} & \multirow{2}{*}{$\begin{array}{c}\% \\
\text { error }\end{array}$} & \multicolumn{2}{|c|}{$\mathrm{R}^{2}$} & \multicolumn{3}{|c|}{$\mathrm{RMSE}^{\mathrm{C}}$} \\
\hline & & & real & predicted & & $\mathrm{C}^{\mathrm{b}}$ & $\mathrm{CV}^{\mathrm{b}}$ & $\mathrm{C}^{\mathrm{b}}$ & $\mathrm{CV}^{\mathrm{b}}$ & $\mathrm{Pb}^{\mathrm{b}}$ \\
\hline \multirow{2}{*}{$\begin{array}{l}\text { milliQ } \\
\text { water }\end{array}$} & $\mathrm{W}, \mathrm{PLS} 2$ & 3 & \multirow[t]{10}{*}{10.30} & 8.73 & 15.2 & 0.997 & 0.995 & 0.852 & 1.082 & 1.111 \\
\hline & W,PLS1 & 3 & & 8.71 & 15.4 & 0.997 & 0.995 & 0.851 & 1.081 & 1.588 \\
\hline \multirow{2}{*}{$\begin{array}{l}\mathrm{NaCl} 0.5 \\
\mathrm{~mol} \mathrm{~L}^{-1}\end{array}$} & W, PLS2 & 2 & & 12.10 & 17.5 & 0.961 & 0.950 & 3.095 & 3.543 & 1.270 \\
\hline & W, PLS1 & 2 & & 11.62 & 12.8 & 0.978 & 0.972 & 2.304 & 2.673 & 1.320 \\
\hline \multirow[t]{2}{*}{ Flix water } & W, PLS2 & 2 & & 10.97 & 6.5 & 0.862 & 0.806 & 5.834 & 6.952 & 0.745 \\
\hline & W, PLS1 & 2 & & 11.29 & 9.6 & 0.896 & 0.852 & 5.055 & 6.057 & 0.992 \\
\hline \multirow{2}{*}{$\begin{array}{l}\text { Deltebre } \\
\text { water }\end{array}$} & L, PLS2 & 2 & & 10.48 & 1.7 & 0.934 & 0.909 & 4.034 & 4.733 & 0.688 \\
\hline & L, PLS1 & 2 & & 10.52 & 2.1 & 0.938 & 0.915 & 3.913 & 4.598 & 0.221 \\
\hline \multirow{2}{*}{$\begin{array}{l}\text { certified } \\
\text { wastewater }\end{array}$} & $\mathrm{L}, \mathrm{PLS} 2$ & 5 & & 10.53 & 2.2 & 0.998 & 0.991 & 0.609 & 1.620 & 0.225 \\
\hline & L, PLS1 & 5 & & 10.22 & 0.8 & 0.999 & 0.992 & 0.401 & 1.524 & 0.085 \\
\hline \multicolumn{11}{|c|}{ Catechol (CC) } \\
\hline \multirow[t]{2}{*}{ Sample } & \multirow{2}{*}{$\begin{array}{l}\text { Baseline }{ }^{a} \text {, } \\
\text { Model }\end{array}$} & \multirow{2}{*}{$\begin{array}{c}\text { Num. } \\
\text { LV }\end{array}$} & \multicolumn{2}{|c|}{$\mathrm{C}_{\mathrm{cC}}(\mathrm{mg} / \mathrm{L})$} & $\%$ & \multicolumn{2}{|c|}{$\mathrm{R}^{2}$} & \multicolumn{3}{|c|}{$\mathrm{RMSE}^{\mathrm{c}}$} \\
\hline & & & real & predicted & error & $\mathrm{C}^{\mathrm{b}}$ & $\mathrm{CV}^{\mathrm{b}}$ & $\mathrm{C}^{\mathrm{b}}$ & $\mathrm{CV}^{\mathrm{b}}$ & $\mathrm{P}^{\mathrm{b}}$ \\
\hline \multirow{2}{*}{$\begin{array}{l}\text { milliQ } \\
\text { water }\end{array}$} & W, PLS2 & 3 & \multirow[t]{10}{*}{15.32} & 15.37 & 0.3 & 0.997 & 0.995 & 0.852 & 1.082 & 1.111 \\
\hline & W, PLS1 & 3 & & 15.37 & 0.3 & 0.997 & 0.995 & 0.818 & 1.029 & 0.053 \\
\hline \multirow{2}{*}{$\begin{array}{l}\mathrm{NaCl} 0.5 \\
\mathrm{~mol} \mathrm{~L}^{-1}\end{array}$} & W, PLS2 & 2 & & 15.31 & 0.1 & 0.992 & 0.982 & 1.306 & 2.081 & 1.270 \\
\hline & W, PLS1 & 2 & & 16.10 & 5.1 & 0.998 & 0.992 & 0.681 & 1.453 & 0.746 \\
\hline \multirow[t]{2}{*}{ Flix water } & W, PLS2 & 2 & & 16.13 & 5.3 & 0.987 & 0.982 & 1.689 & 2.067 & 0.745 \\
\hline & W, PLS1 & 2 & & 16.18 & 5.6 & 0.976 & 0.967 & 2.307 & 2.741 & 0.862 \\
\hline \multirow{2}{*}{$\begin{array}{l}\text { Deltebre } \\
\text { water }\end{array}$} & L, PLS2 & 2 & & 14.36 & 6.3 & 0.996 & 0.994 & 0.882 & 1.135 & 0.688 \\
\hline & L, PLS1 & 2 & & 14.40 & 6.0 & 0.997 & 0.995 & 0.846 & 1.087 & 0.920 \\
\hline \multirow{2}{*}{$\begin{array}{l}\text { certified } \\
\text { wastewater }\end{array}$} & $\mathrm{L}, \mathrm{PLS} 2$ & 5 & & 15.54 & 1.4 & 1.000 & 0.998 & 0.215 & 0.736 & 0.225 \\
\hline & L, PLS1 & 5 & & 15.32 & 0.0 & 1.000 & 0.998 & 0.223 & 0.659 & 0.228 \\
\hline
\end{tabular}

a) W=Whittaker; L=Automatic weighted least squares (AWLS);

b) $\mathrm{C}=$ calibration, $\mathrm{CV}=$ Cross-validation, $\mathrm{P}=$ prediction;

c) RMSE=root mean squares error 


\section{FIGURE CAPTIONS}

Figure 1

Calibration plots of univariate (a) and multivariate (b) standard addition using voltammetric experiments, with $\mathrm{E}, \mathrm{I}, \mathrm{I}_{\mathrm{p}}, \mathrm{C}_{\mathrm{add}}$ being the potential, the current, the peak current and the added concentration of analyte. The sample is denoted by a black point or a thick line. The comparison between the true added concentrations and these predicted by the calibration model is shown in (c). For more details, see explanation in the text.

Figure 2

Different strategies to carry out calibration measurements: addition of a solution containing known concentrations of two analytes A and B when they produce separated (a) and overlapped signals (b); addition of two separated solutions, one for every analyte, in an alternating (c) or in an analyte-wise way (d).

Figure 3

Voltammograms corresponding to the determination of hydroquinone (HQ) and catechol (CC) in different matrices: a) Buffer only; b) $\mathrm{NaCl} 0.5 \mathrm{~mol} \mathrm{~L}^{-1}$ solution; c) Ebro River water, Flix location; d) Ebro River water, Deltebre location; e) certified waste water. All experiments were done in a PBS buffer media at $\mathrm{pH}$ 7. Voltammograms of the respective blanks are shown in black, samples are depicted in green and alternating additions of $\mathrm{HQ}$ and $\mathrm{CC}$ are denoted by red and blue colours.

Figure 4

Effects on a set of original differential pulse voltammograms (a) of the baseline correction according to the Whittaker method (b) or to the automatic weighted least squares (AWLS) method (c).

Figure 5

Predicted added concentrations of hydroquinone (a) and catechol (b) in the certified wastewater sample according to PLS multivariate standard addition methodology as a function of the real spiked concentrations. The original sample is placed just in the origin of 
coordinates, whereas the successive additions are depicted as black points. The blank, i.e., the target of extrapolation, is denoted in red. The green line is the ideal prediction with slope one and zero intercept. Parameters informing about the reliability of PLS models are also shown in the insets. In this case, two PLS1 models have been used, one for each analyte. 
Figure 1

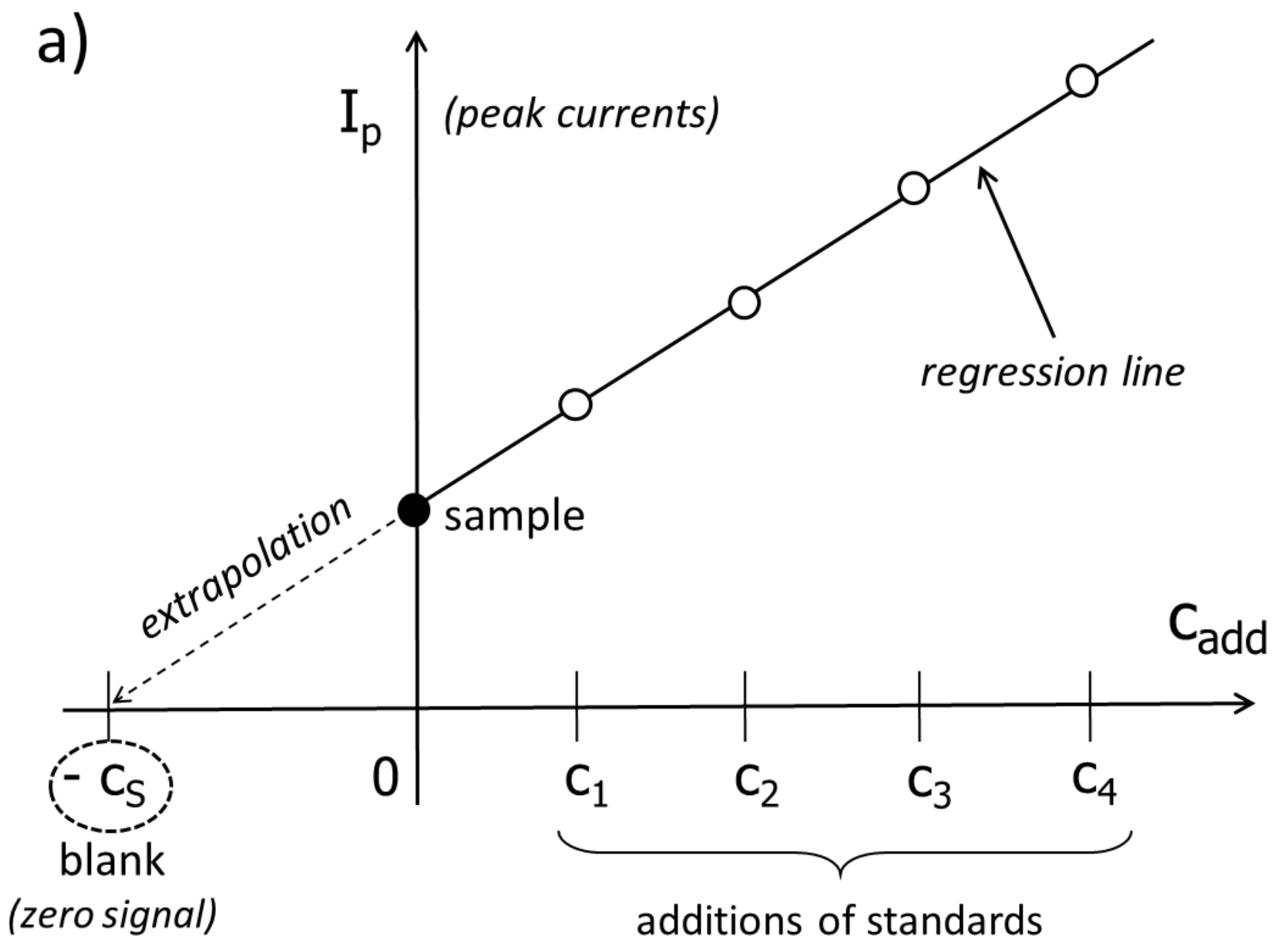


Figure 1

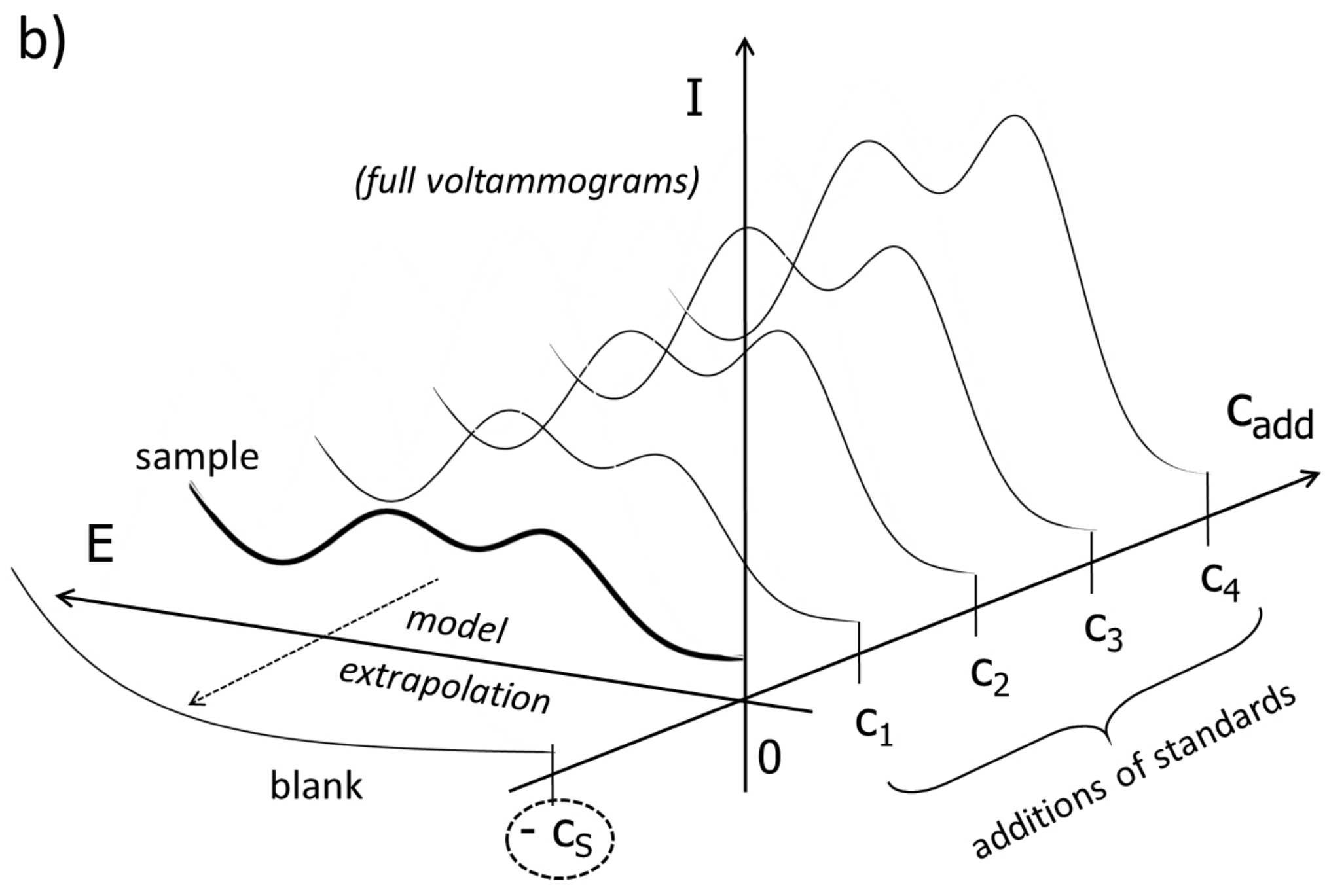


Figure 1

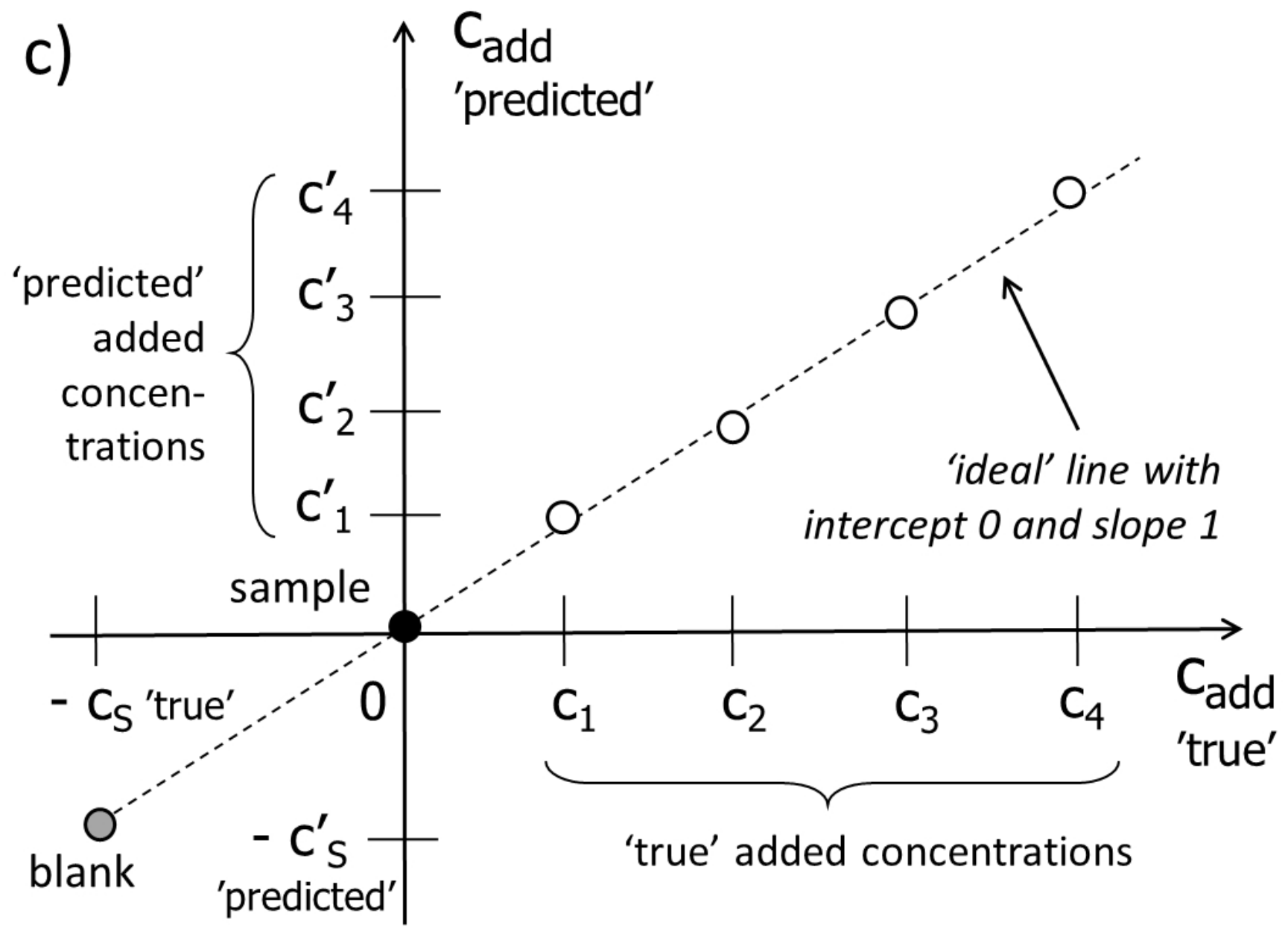


Figure 2

a)

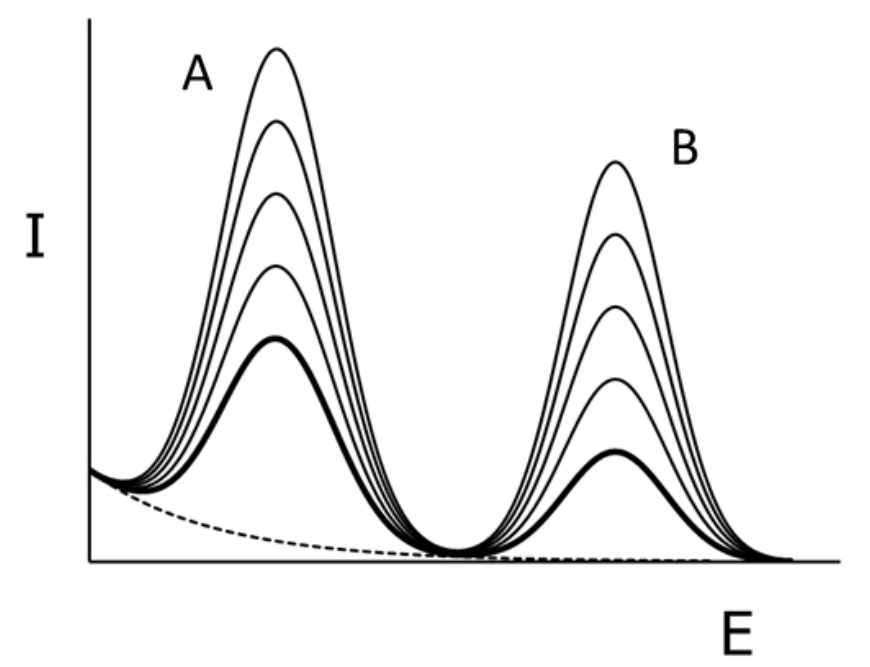

c)

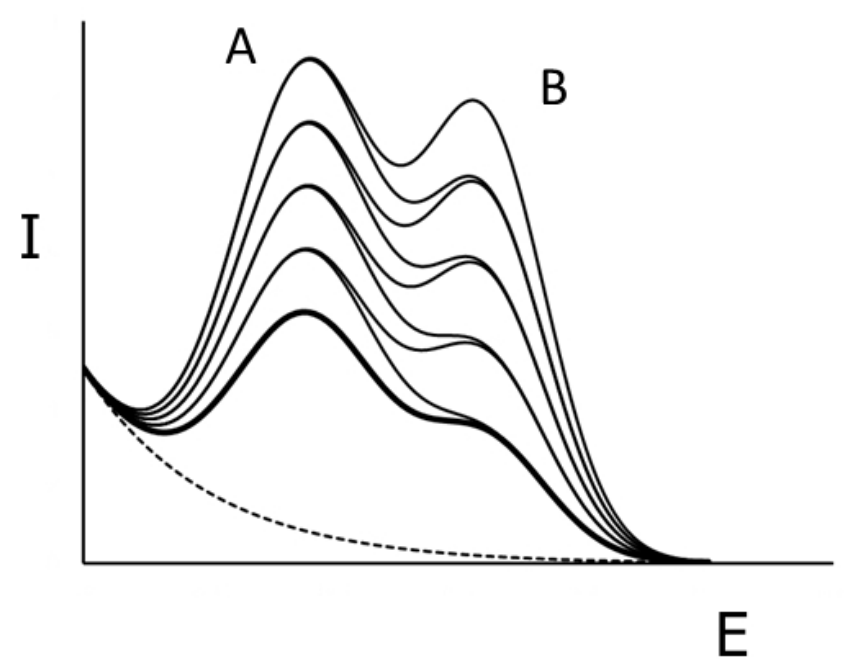

b)

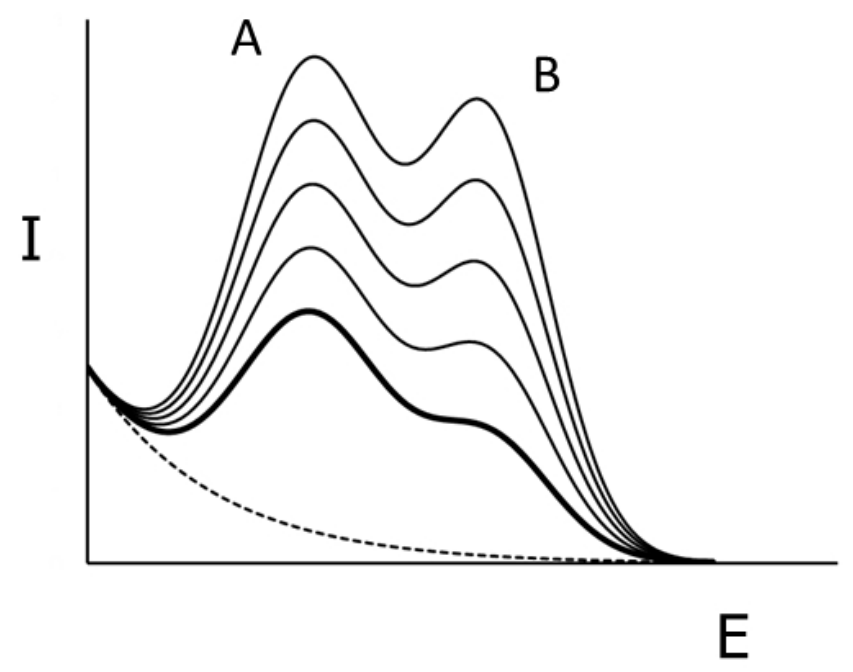

d)

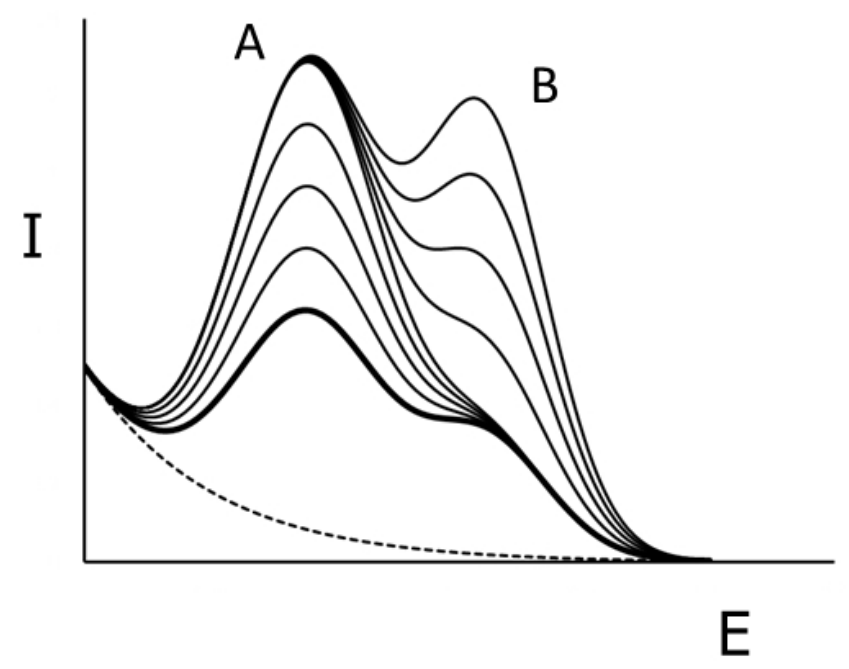


Figure 3
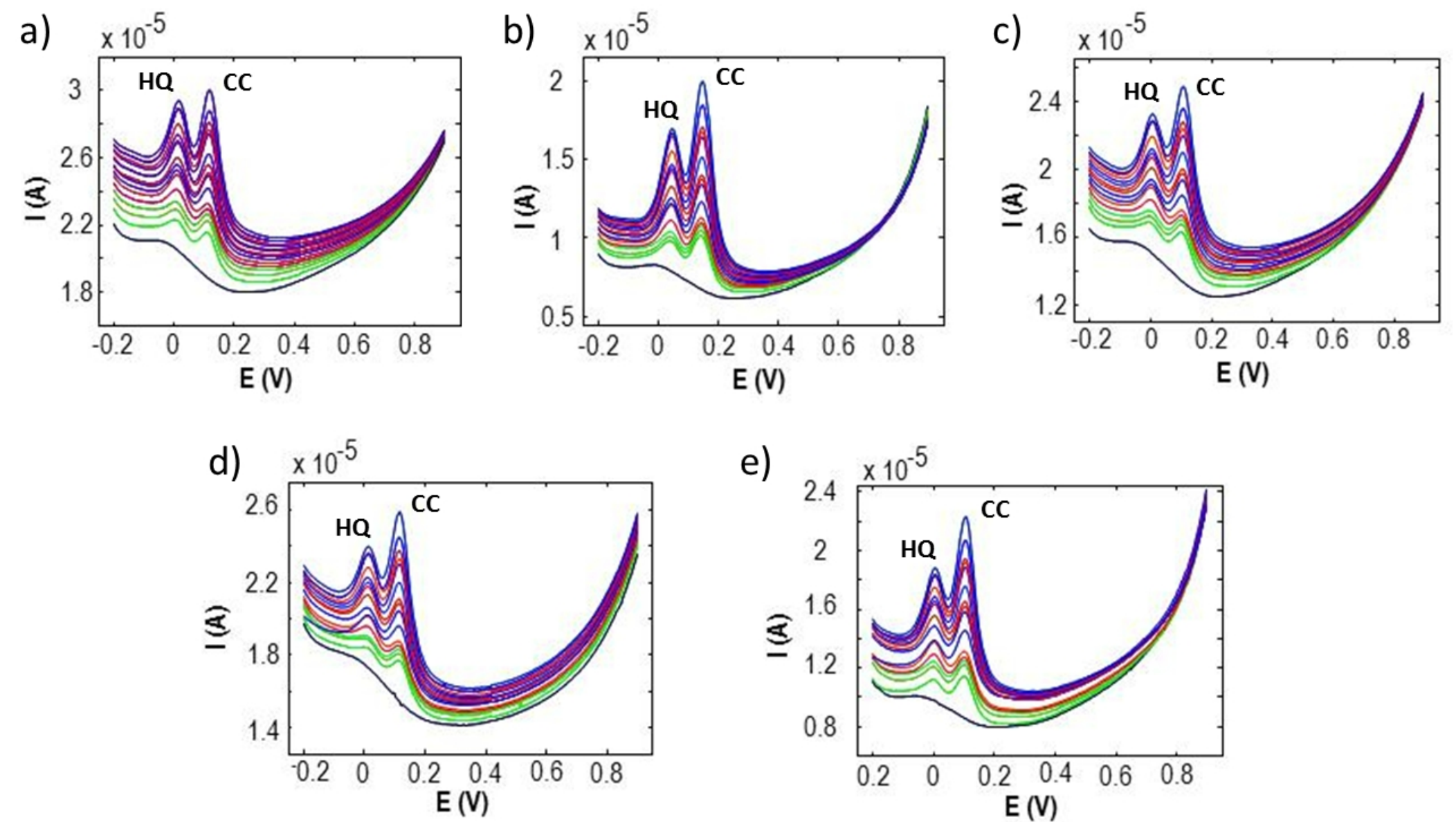
Figure 4

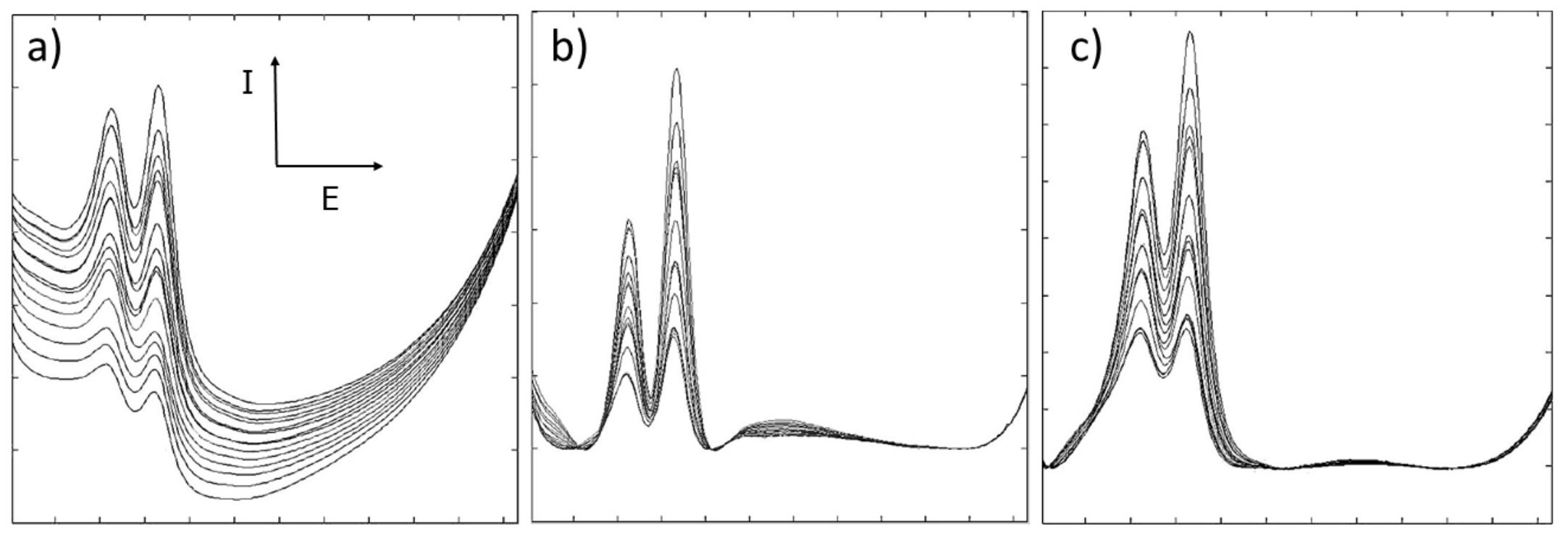


Figure 5a

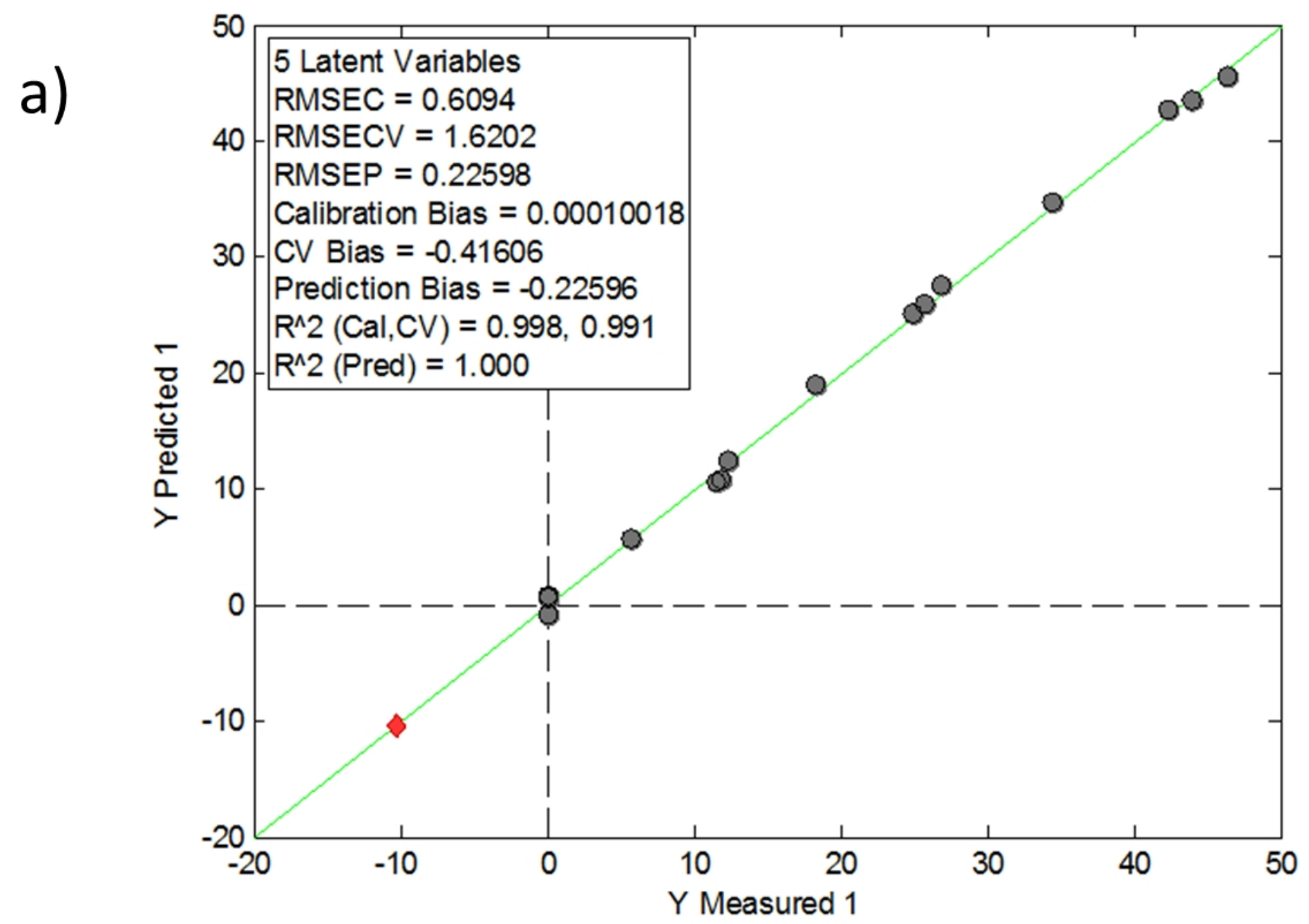


Figure 5b

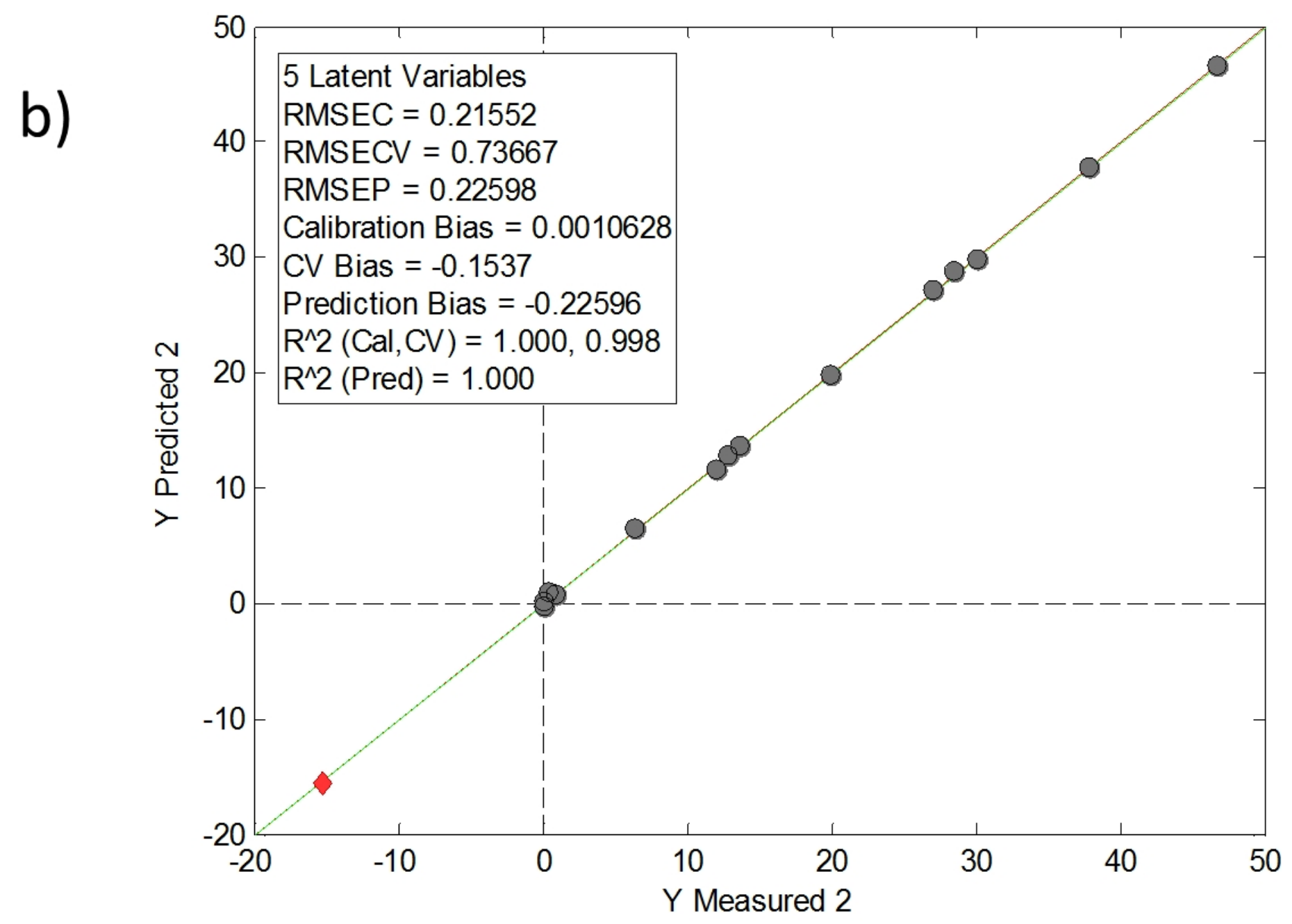




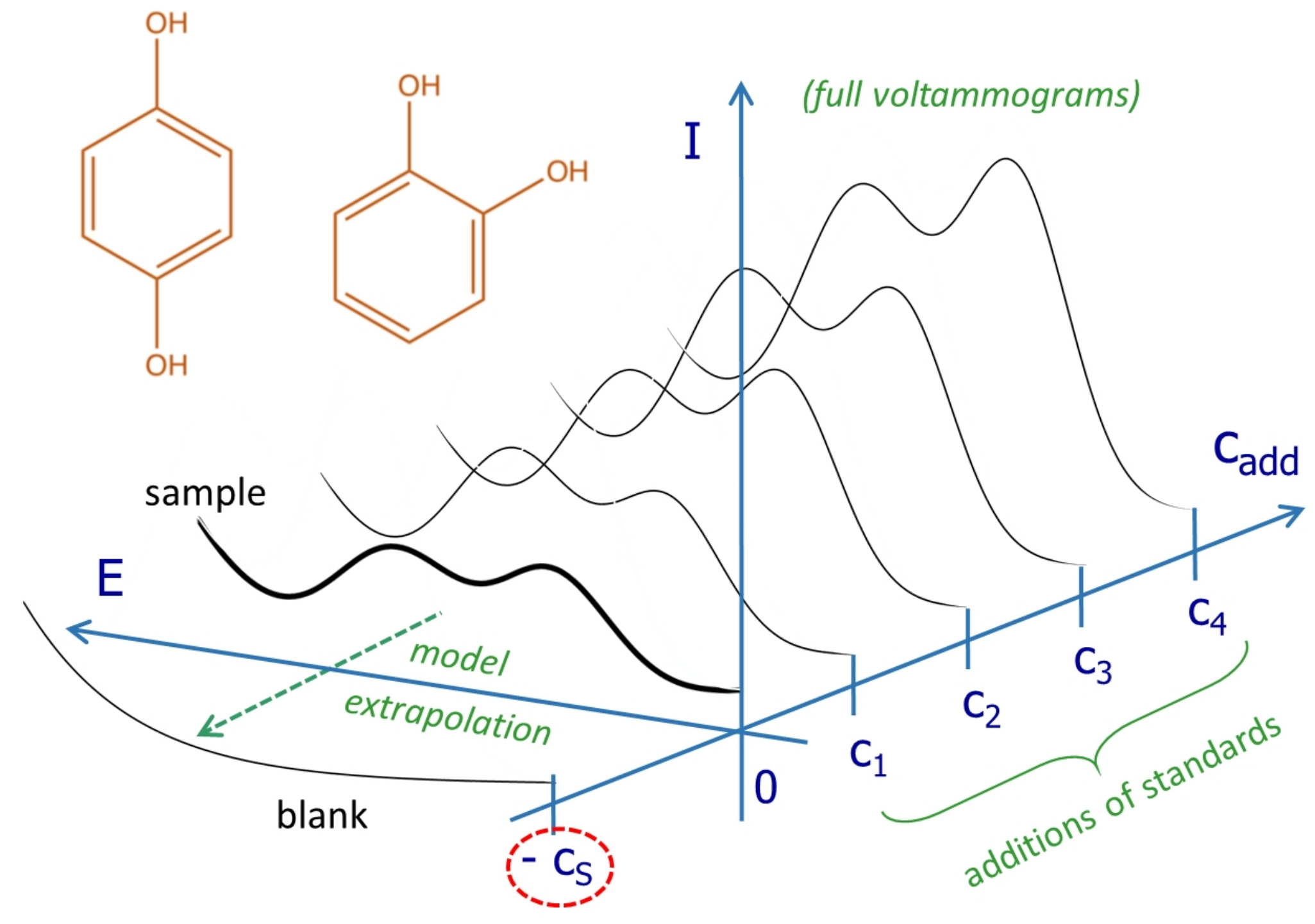

